\title{
Model predictive control of bidirectional isolated DC-DC converter for energy conversion system
}

\author{
Parvez Akter ${ }^{\mathrm{a}}$, Muslem Uddin ${ }^{\mathrm{a}}$, Saad Mekhilef ${ }^{\mathrm{a} *}$, Nadia Mei Lin Tan ${ }^{\mathrm{b}}$ \\ and Hirofumi Akagi ${ }^{\mathrm{c}}$ \\ ${ }^{a}$ Power Electronics and Renewable Energy Research Laboratory (PEARL), Department of \\ Electrical Engineering, University of Malaya, Kuala Lumpur 50603, Malaysia; ${ }^{b}$ Department of \\ Electrical Power Engineering, Universiti Tenaga Nasional, Kajang 43000, Malaysia; ${ }^{\circ}$ Department \\ of Electrical and Electronic Engineering, Tokyo Institute of Technology, Tokyo 152-8552, Japan
}

(Received 10 September 2013; accepted 27 September 2014)

\begin{abstract}
Model predictive control (MPC) is a powerful and emerging control algorithm in the field of power converters and energy conversion systems. This paper proposes a model predictive algorithm to control the power flow between the high-voltage and lowvoltage DC buses of a bidirectional isolated full-bridge DC-DC converter. The predictive control algorithm utilises the discrete nature of the power converters and predicts the future nature of the system, which are compared with the references to calculate the cost function. The switching state that minimises the cost function is selected for firing the converter in the next sampling time period. The proposed MPC bidirectional DC-DC converter is simulated with MATLAB/Simulink and further verified with a $2.5 \mathrm{~kW}$ experimental configuration. Both the simulation and experimental results confirm that the proposed MPC algorithm of the DC-DC converter reduces reactive power by avoiding the phase shift between primary and secondary sides of the high-frequency transformer and allow power transfer with unity power factor. Finally, an efficiency comparison is performed between the MPC and dualphase-shift-based pulse-width modulation controlled DC-DC converter which ensures the effectiveness of the MPC controller.
\end{abstract}

Keywords: predictive control; bidirectional DC-DC converter; unity power factor; reactive power; power conversion system

\section{Introduction}

An efficient bidirectional DC-DC converter is indispensable to manage the power flow by switching action to provide high performance and efficiency of an energy conversion system. Hence, the control algorithm of this DC-DC converter needs to be immensely effective as it consists of two conversion stages (single-phase inverter and rectifier) along with an isolated high-frequency transformer (Zhao, Song, Liu, \& Sun, 2014). Several improved control techniques, such as fuzzy-neural control (Cheng, Hsu, Lin, Lee, \& Li, 2007), hysteresis control (Leung \& Chung, 2005) and sliding-mode control (Cheng et al., 2007; Tsai \& Chen, 2007), have been investigated in power electronic systems to control power converters. The practical applications of these control methods are confined to simple configured boost, buck, half-bridge and full-bridge unidirectional converters topologies till today. Nevertheless, these controls are aimed to control more complex-

*Corresponding author. Email: saad@um.edu.my 
configured converters topologies (Bai \& Mi, 2008a; Rivetta, Emadi, Williamson, Jayabalan, \& Fahimi, 2006).

The bidirectional isolated DC-DC converter has many advantages over traditional converters, such as bidirectional energy flow, soft switching, galvanic isolation, highpower density and low-system parasitic sensitivity as has been investigated in Bai and Mi (2008b), Tan, Abe, and Akagi (2012) and Naayagi, Forsyth, and Shuttleworth (2012). A traditional PI-based phase-shift control has been used previously to control the bidirectional isolated DC-DC converter in DeDoncker, Kheraluwala, and Divan (1991). This method of control has a simple structure and is very easy to implement. Moreover, a phase-shift modulation control has been applied for high-power transfer in Kheraluwala, Gascoigne, Divan, and Baumann (1992) and Tan, Inoue, Kobayashi, and Akagi (2008). The major drawbacks of this phase-shift modulation control are reactive power losses and existence of dead-time effect. Subsequently, various control algorithms have been introduced to improve the performance of the system. In order to achieve a higher converter efficiency and expand the zero-voltage switching region, a phase-shift plus pulse-width modulation (PWM) algorithm has been applied in Xu, Zhao, and Fan (2004) and the effectiveness is verified for soft switching in Lee, Ko, and Chi (2010) and Xiao and Xie (2008). On the other hand, in literature a dualphase-shift (DPS) control method is proposed to eliminate the reactive power in isolated bidirectional full-bridge DC-DC converter (Bai \& Mi, 2008b), which is being extended as a model-based phase-shift control and applied in Bai, Nie, and Mi (2010) to improve dynamic performance of the converter. Furthermore, two PI controller-based adaptive control algorithm is presented in De Breucker, Engelen, Tant, and Driesen (2010) to operate and verify the changes of current in small and large extent separately. There is a trade-off between transient response and robustness in parameter selection of a PI controller, which is very empirical. Therefore, computeraided optimisation of DC-DC converter has been carried out by using two PWM controller integrated circuits in Neugebauer and Perreault (2003) and Garcia, Zumel, de Castro, and Cobos (2006).

The principal feature of the model predictive control (MPC) scheme is to predict the future behaviour of control variables. This control algorithm has become the most attractive mode of technique to control the bidirectional DC-DC converter comparing with all the classical control techniques discussed above due to its simple and intuitive concept with fast dynamic responses (Cortes, Kazmierkowski, Kennel, Quevedo, \& Rodriguez, 2008; Rodriguez et al., 2013). Moreover, the MPC algorithm is easy to configure with constraints and non-linearity and also very easy for practical implementation. The fast and powerful microprocessors are available today to implement the predictive control algorithm very easily as it requires higher number of calculations compared with all the classical controls stated in Cortes et al. (2008) and Muslem Uddin, Mekhilef, Rivera, and Rodriguez (2013). So, the application of the MPC algorithm in power converter is increasing day by day. To date, this algorithm is proposed for a DC-DC buck converter (Bibian \& Jin, 2002; Geyer, Papafotiou, \& Morari, 2008), boost converter (Bibian \& Jin, 2001) and buck-boost converter (Chen, Prodic, Erickson, \& Maksimovic, 2003). Xie, Ghaemi, Sun, and Freudenberg (2012) proposed MPC for full-bridge DC-DC converter. However, this model is limited only for unidirectional power flow. Although model predictive algorithm is an efficient and attractive alternative for controlling the power converters, it has not been used to control power flow of a bidirectional isolated DC-DC converter yet. 
This paper proposes an MPC algorithm and its application for a bidirectional isolated full-bridge DC-DC converter and is organised in the following manner. The system configuration and working principle of energy conversion system topology are elaborately described in Section 2. The formulation of the MPC method with discrete time model, the cost function used for selection of the switching state and a detailed explanation of the control scheme and algorithm are mentioned in Section 3. The efficiency and performance of the proposed MPC bidirectional isolated DC-DC converter is tested with MATLAB/ Simulink, and the simulation results are analysed in Section 4. Therefore, the DC-DC converter is further verified with a $2.50 \mathrm{~kW}$ experimental set-up, which is depicted in Section 5. Finally, the conclusions are drawn in Section 6.

\section{Description of energy conversion system topology}

\subsection{System configuration}

Figure 1 shows the energy conversion system topology, which bidirectionally converts the $\mathrm{AC}$ power from three-phase AC grid to low-voltage $\mathrm{DC}$ bus and is configured with a three-phase bidirectional PWM controlled AC-DC converter and a bidirectional fullbridge isolated DC-DC converter. The energy conversion system in Figure 1 is similar to that in Tan et al. (2012) because this paper intends to improve the efficiency and performance of the bidirectional isolated DC-DC converter using the MPC algorithm.

The bidirectional DC-DC converter consists of two symmetrical structured converters denoted as bridge 1 and bridge 2, which are isolated with a high-frequency $(4 \mathrm{kHz})$ transformer. Bridge 1 consists of four IGBT-Diode switches $\left(S_{1}-S_{4}\right)$. Each leg of the converter contains two IGBTs in series. A snubber capacitor is connected with each of the IGBTs for minimising the turn-off overvoltage and also achieves zero-voltage switching. Again, bridge 2 is configured with four MOSFET switches $\left(S_{5}-S_{8}\right)$, as it is operated in low-voltage $(60 \mathrm{~V})$ condition. To minimise the switching loss, small snubber capacitor is connected with each MOSFET switches.

\subsection{Working principle}

The MPC algorithm is applied to control the power flow of the bidirectional isolated DC-DC converter. The working principle of the MPC method is based on a finite number

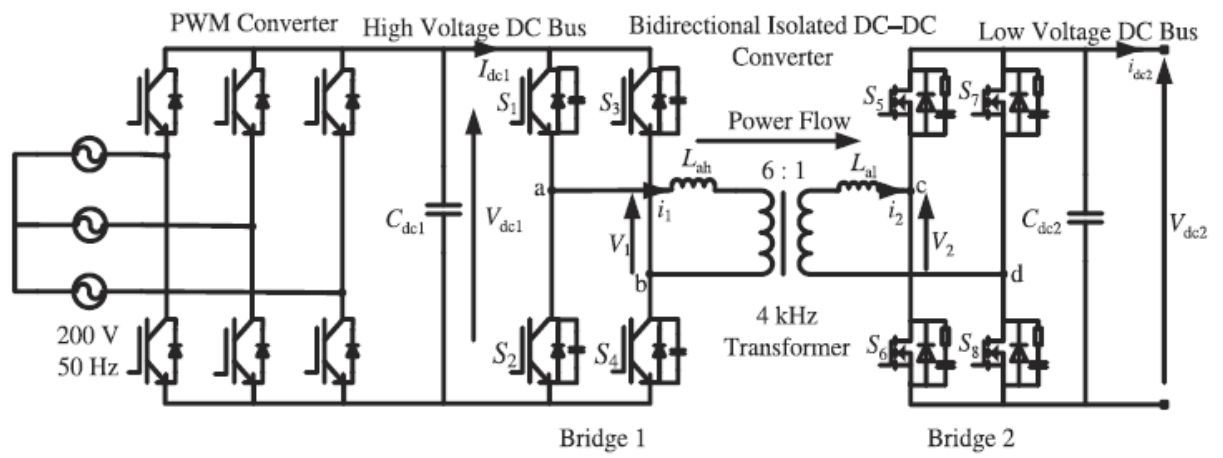

Figure 1. Energy conversion system based on the bidirectional isolated DC-DC converter (Tan et al., 2012). 




Figure 2. Simplified bidirectional isolated high-frequency transformer linked DC-DC converter scheme.

of possible switching states, which utilises the discrete behaviour of a static power converter. In the case of the bidirectional isolated DC-DC converter, the MPC algorithm utilises the discrete nature of equivalent inductances $\left(L_{\mathrm{eq}}\right)$ to control the power flow by appropriate switching action. The equivalent inductance $\left(L_{\mathrm{eq}}\right)$ is defined with the simplified diagram of the bidirectional DC-DC converter presented in Figure 2, as the equivalent inductance value of the transformer high-side auxiliary inductance $\left(L_{\mathrm{ah}}\right)$, low-side auxiliary inductance $\left(L_{\mathrm{al}}\right)$ and leakage inductance $\left(L_{\text {leak }}\right)$. For the selection of the appropriate switching state to be applied to the converter, a selection criterion must be defined with a cost function which measures the error between the references and the predicted values. Finally, the state that minimises the cost function is selected for the next sampling interval.

The gating signals $S_{\mathrm{a}}, S_{\mathrm{b}}, S_{\mathrm{c}}$ and $S_{\mathrm{d}}$ determine the switching states of the bidirectional DC-DC converter as follows:

$$
\begin{aligned}
& S_{\mathrm{a}}=\left\{\begin{array}{l}
1, S_{1} \text { is on and } S_{2} \text { is off } \\
0, S_{1} \text { is off and } S_{2} \text { is on }
\end{array}\right. \\
& S_{\mathrm{b}}=\left\{\begin{array}{l}
1, S_{3} \text { is on and } S_{4} \text { is off } \\
0, S_{3} \text { is off and } S_{4} \text { is on }
\end{array}\right. \\
& S_{\mathrm{c}}=\left\{\begin{array}{l}
1, S_{5} \text { is on and } S_{6} \text { is off } \\
0, S_{5} \text { is off and } S_{6} \text { is on }
\end{array}\right. \\
& S_{\mathrm{d}}= \begin{cases}1, S_{7} \text { is on and } S_{8} \text { is off } \\
0, S_{7} \text { is off and } S_{8} \text { is on }\end{cases}
\end{aligned}
$$

Hence, the switching function $(S)$ for bridges 1 and 2 of the DC-DC converter can be expressed as

$$
S_{\text {bridgel }}=S_{\mathrm{a}}-S_{\mathrm{b}}
$$

and

$$
S_{\text {bridge } 2}=S_{\mathrm{c}}-S_{\mathrm{d}}
$$

The bidirectional DC-DC converter operates in two modes. The first is the buck mode, which allows power transfer from high-voltage DC bus to low-voltage DC bus. 




Figure 3. Buck mode operation of the DC-DC converter.

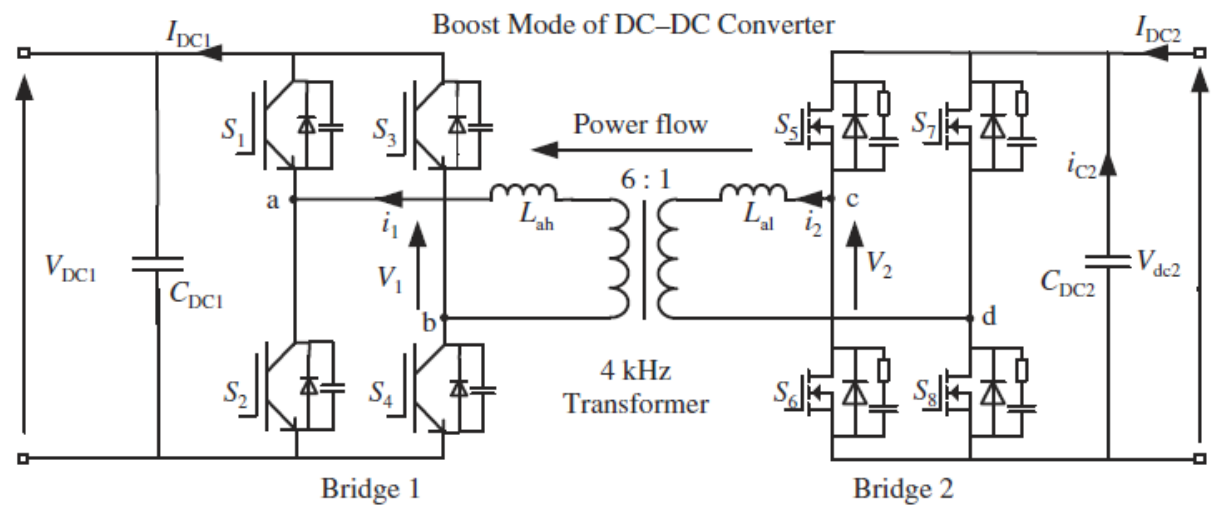

Figure 4. Boost mode operation of the DC-DC converter.

During this buck mode operation, bridge 1 of the bidirectional isolated DC-DC converter (Figure 3) works as a DC-AC inverter, supplying power to high-frequency transformer, while bridge 2 works as an $\mathrm{AC}-\mathrm{DC}$ rectifier. On the other hand, in case of the boost mode, power flows in the opposite direction and bridge 2 of the DC-DC converter (Figure 4) act as a DC-AC inverter and bridge 1 as an $\mathrm{AC}-\mathrm{DC}$ rectifier.

\subsubsection{Buck mode operation}

Buck mode operation of the DC-DC converter with the MPC method is illustrated in Figure 3, where bridge 1 of the DC-DC converter acts as an inverter and the equivalent inductance $L_{\text {eq }}$, resistance $R_{\text {eq }}$ and rest of the part of the circuit act as an equivalent load for the inverter. By applying Kirchhoff's voltage law at the AC side of inverter (bridge 1), the model current equation of the inverter becomes

$$
L_{\mathrm{eq}} \frac{d i_{1}}{d t}=S_{\text {bridge1 }} V_{\mathrm{dc} 1}-n V_{2}
$$


where $L_{\mathrm{eq}}$ is the sum of transformer leakage inductance ( $\left.L_{\text {leak }}\right), L_{\mathrm{ah}}$ and $n^{2} L_{\mathrm{al}}$ mentioned in Figure 3. $V_{\mathrm{dcl}}$ is the $\mathrm{DC}$ voltage at high-voltage $\mathrm{DC}$ bus acting as supply voltage for bridge 1 .

On the other hand, bridge 2 of the converter works as a single-phase voltage mode controlled rectifier. The transformer secondary winding voltage $\left(V_{2}\right)$ and current $\left(i_{2}\right)$ act as the source voltage and current for this rectifier. Therefore, the model voltage equation of the rectifier can be obtained by using the Kirchhoff's current law at the low-voltage DC bus side

$$
C_{\mathrm{dc} 2} \frac{d V_{\mathrm{dc} 2}}{d t}=i_{2} S_{\text {bridge } 2}-I_{\mathrm{dc} 2}
$$

where $C_{\mathrm{dc} 2}$ is the capacitance connected in parallel with low-voltage DC bus; $V_{\mathrm{dc} 2}$ and $I_{\mathrm{dc} 2}$ are the DC voltage and DC current respectively at the low-voltage DC bus.

\subsubsection{Boost mode operation}

Figure 4 describes the boost mode operation of the DC-DC converter, in which the energy is transferred from low-voltage DC bus to high-voltage DC bus. In this case, bridge 2 of the DC-DC converter acts as an inverter and bridge 1 as a rectifier. Hence, similar to Equations (7) and (8), the model of the inverter and rectifier in the boost mode can be obtained and presented in Equations (9) and (10):

$$
L_{\text {eq }} \frac{d i_{2}}{d t}=S_{\text {bridge } 2} V_{\text {dc2 }}-\frac{V_{1}}{n}
$$

where $V_{\mathrm{dc2} 2}$ is the $\mathrm{DC}$ voltage at low-voltage $\mathrm{DC}$ bus acting as supply voltage for bridge 2 .

$$
C_{\mathrm{dc} 1} \frac{d V_{\mathrm{dc} 1}}{d t}=i_{1} S_{\mathrm{bridge} 1}-I_{\mathrm{dc} 1}
$$

where $C_{\mathrm{dcl}}$ is the capacitance connected in parallel with high-voltage DC bus; $V_{\mathrm{dcl}}$ and $I_{\mathrm{dcl}}$ are the DC voltage and DC current respectively at the high-voltage DC bus.

\section{Formulation of the MPC method for the DC-DC converter}

The formulation of the MPC algorithm for the bidirectional isolated DC-DC converter is described in the following section. It is necessary to transform the dynamic system of the DC-DC converter for both buck and boost mode operations represented in Equations (7)(10) into discrete time model at a specific sampling time $T_{\mathrm{S}}$.

\subsection{Discrete time model for prediction horizon}

The discrete time model is used to predict the future values of currents and voltages in the next sampling interval $(k+1)$, from the measured currents and voltages at the $k$ th sampling instant. The system model derivative $\frac{d x}{d t}$ from Euler approximation can be expressed as 


$$
\frac{d x}{d t} \approx \frac{x(k+1)-x(k)}{T_{\mathrm{s}}}
$$

Using the above approximation, the discrete time model of predictive currents and voltages for the next $(k+1)$ sampling instant of the bidirectional full-bridge DC-DC converter in the buck and boost modes can be derived.

\subsubsection{Buck mode}

During the buck mode operation of the system, bridge 1 of the DC-DC converter works as an inverter and is controlled in current mode. On the other hand, bridge 2 is controlled with voltage mode as it works as a rectifier. Hence, the discrete time model of predictive currents at the next sampling instant $(k+1)$ for the inverter (bridge 1) of the DC-DC converter can be evaluated from Equations (7) and (11) as

$$
i_{1}(k+1)=i_{1}(k)+\frac{T_{\mathrm{s}}}{L_{\mathrm{eq}}}\left\{S_{\text {bridgel }} V_{\mathrm{dc} 1}(k)-n V_{2}(k)\right\}
$$

and the discrete time model of predictive voltage at the next sampling instant $(k+1)$ for the rectifier (bridge 2) can also be presented from Equations (8) and (11) as

$$
V_{\mathrm{dc} 2}(k+1)=V_{\mathrm{dc} 2}(k)+\frac{T_{\mathrm{s}}}{C_{\mathrm{dc} 2}}\left\{S_{\text {bridge } 2} i_{2}(k)-I_{\mathrm{dc} 2}(k)\right\}
$$

\subsubsection{Boost mode}

In the boost mode operation, the DC-DC converter operates in reverse mode corresponding to the buck mode. Therefore, bridge 1 of this converter works as a rectifier and bridge 2 as an inverter. Then, the discrete time model of predictive voltage and current for the inverter and rectifier can be written as

$$
V_{\mathrm{dc} 1}(k+1)=V_{\mathrm{dcl}}(k)+\frac{T_{\mathrm{s}}}{C_{\mathrm{dcl}}}\left\{S_{\text {bridgel }} i_{1}(k)-I_{\mathrm{dcl}}(k)\right\}
$$

and

$$
i_{2}(k+1)=i_{2}(k)+\frac{T_{\mathrm{s}}}{L_{\mathrm{eq}}}\left\{S_{\text {bridge } 2} V_{\mathrm{dc} 2}(k)-\frac{V_{1}(k)}{n}\right\}
$$

\subsection{Cost function}

The main objective of the MPC algorithm is to minimise the error with fast dynamic response between the predicted and reference values of the discrete variables. To achieve this objective, an appropriate cost function is defined with a measurement of predicted input error. Hence, the cost function for inverter and rectifier can be expressed with the absolute error between the predictive and reference values for both buck and boost modes as follows: 
Link to full text journal article :

http://www.tandfonline.com/doi/pdf/10.1080/00207217.2015.1028479 